\title{
RECONSTRUCTION OF GRADED GROUPOIDS FROM GRADED STEINBERG ALGEBRAS
}

\author{
PERE ARA, JOAN BOSA, ROOZBEH HAZRAT, AND AIDAN SIMS
}

\begin{abstract}
We show how to reconstruct a graded ample Hausdorff groupoid with topologically principal neutrally graded component from the ring structure of its graded Steinberg algebra over any commutative integral domain with 1 , together with the embedding of the canonical abelian subring of functions supported on the unit space. We deduce that diagonal-preserving ring isomorphism of Leavitt path algebras implies $C^{*}$ isomorphism of $C^{*}$-algebras for graphs $E$ and $F$ in which every cycle has an exit.
\end{abstract}

\section{INTRODUCTION}

Since the independent introduction of Leavitt path algebras by Abrams-Aranda-Pino [1] and by Ara-Moreno-Pardo [5], there has been a great deal of interest in the many parallels between the theory of Leavitt path algebras and that of graph $C^{*}$-algebrasparticularly because the reasons for these parallels are frequently not apparent from the standard generators-and-relations picture. A key unresolved conjecture, due to Abrams and Tomforde, says that if $E$ and $F$ are graphs whose complex Leavitt path algebras are isomorphic as rings, then they have isomorphic $C^{*}$-algebras (see [30, Question 6]). In this paper, we make some progress on this question by studying diagonal-preserving isomorphisms of Steinberg algebras. We confirm a slight weakening of Abrams and Tomforde's conjecture: if $E$ and $F$ are graphs in which every cycle has an exit and there is a ring-isomorphism $L_{\mathbb{C}}(E) \cong L_{\mathbb{C}}(F)$ that respects the canonical diagonal, then there is a diagonal-preserving isomorphism $C^{*}(E) \cong C^{*}(F)$.

Our approach uses Steinberg algebras, introduced in [27] and, independently, in [8]. The groupoid $C^{*}$-algebra of a groupoid $\mathcal{G}$ is a norm completion of the convolution algebra of continuous, compactly supported functions from $\mathcal{G}$ to $\mathbb{C}$. When $\mathcal{G}$ is totally disconnected, the Steinberg algebra $A_{R}(\mathcal{G})$ for a ring $R$ is the convolution algebra of locally constant functions from $\mathcal{G}$ to $R$; so $A_{\mathbb{C}}(\mathcal{G})$ is a dense subalgebra of $C^{*}(\mathcal{G})$. In particular, when $\mathcal{G}$ is the graph groupoid [17] of a directed graph $E$, so that $C^{*}(\mathcal{G})$ is the graph algebra $C^{*}(E)$, the Steinberg algebras $A_{R}(\mathcal{G})$ are precisely the Leavitt path $R$-algebras $L_{R}(E)$ (see [8, Remark 4.4] and [9, Example 3.2]).

The algebra $C_{0}\left(\mathcal{G}^{(0)}\right)$ of continuous complex-valued functions vanishing at infinity on $\mathcal{G}^{(0)}$ is a commutative $C^{*}$-subalgebra of $C^{*}(\mathcal{G})$, and the algebra $D$ of locally constant functions from $\mathcal{G}^{(0)}$ to $R$ is a commutative subalgebra of each $A_{R}(\mathcal{G})$. In [26] (see also [14]), Renault showed that if $\mathcal{G}$ is topologically principal, then it can be reconstructed from the data $\left(C^{*}(\mathcal{G}), C_{0}\left(\mathcal{G}^{(0)}\right)\right)$. So if $\mathcal{G}$ and $\mathcal{H}$ are topologically principal groupoids,

Date: May 10, 2018.

The first and second-named authors were partially supported by the grants DGI MICIIN MTM201128992-C02-01 and MINECO MTM2014-53644-P. The second author is supported by the Beatriu de Pinós fellowship (2014 BP-A 00123). This research was supported by Australian Research Council grant DP150101598. 
then there is an isomorphism $C^{*}(\mathcal{G}) \cong C^{*}(\mathcal{H})$ that carries $C_{0}\left(\mathcal{G}^{(0)}\right)$ to $C_{0}\left(\mathcal{H}^{(0)}\right)$ if and only if $\mathcal{G} \cong \mathcal{H}$. These results have been used recently to prove remarkable results about continuous orbit equivalence rigidity for symbolic dynamical systems [7, 21, 22].

In this paper, we prove that if $\mathcal{G}$ is ample and Hausdorff, $c: \mathcal{G} \rightarrow \Gamma$ is a 1-cocycle taking values in a discrete group, $c^{-1}(0)$ is a topologically principal subgroupoid of $\mathcal{G}$, and $R$ is a commutative integral domain with 1 , then $D$ is a maximal commutative subring of the ring $A_{R}(\mathcal{G})$, and we can recover $\mathcal{G}$ from the pair $\left(A_{R}(\mathcal{G}), D\right)$ regarded as a $\Gamma$-graded ring with distinguished commutative subring. As a direct consequence, we deduce the following. Suppose that $E$ and $F$ are directed graphs in which every cycle has an exit, and suppose that there is a commutative integral domain $R$ with 1 for which there is a ring isomorphism $\pi: L_{R}(E) \cong L_{R}(F)$ that such $\pi\left(s_{\mu} s_{\mu^{*}}\right) s_{\eta} s_{\eta^{*}}=s_{\eta} s_{\eta^{*}} \pi\left(s_{\mu} s_{\mu^{*}}\right)$ for every path $\mu$ in $E$ and every path $\eta$ in $F$. Then there is a diagonal-preserving isomorphism $C^{*}(E) \cong C^{*}(F)$. We also make a little progress on the vexing question [30, Question 1]: are the Leavitt path algebras $L_{2, K}$ and $L_{2-, K}$ isomorphic for a field $K$ ? Our results imply that there is no diagonal-preserving ring-isomorphism between these two algebras.

A similar result about Leavitt path algebras, of which we became aware late in the preparation of this work, was obtained recently by Brown-Clark-an Huef [6]. Neither our result nor theirs is a direct generalisation of the other, though: their theorem requires a ${ }^{*}$-ring isomorphism and that $E$ be row-finite with no sinks (no sources with their conventions), whereas ours requires only a ring isomorphism and does not insist that $E$ should be row-finite or have no sinks; but our result requires that every cycle in $E$ have an exit whereas theirs does not. But our result has many further applications; for example, to Kumjian-Pask algebras of higher-rank graphs, to algebras associated to Cantor minimal systems, to the algebras $L^{a b}(E, C)$ associated to separated graphs by Ara and Exel in [4, and to all groupoids arising from partial actions of countable discrete groups on totally disconnected metrisable spaces [11]. Indeed, by [27, Theorems 5.17 and 6.3], our result applies to all algebras associated to inverse semigroups that are weak semilattices.

Acknowledgement. We are very grateful to the anonymous referee, whose helpful comments have significantly improved the exposition of the paper, and have also suggested interesting lines of further enquiry.

\section{Preliminaries}

2.1. Groupoids and Inverse Semigroups. We give a very brief introduction to Hausdorff ample groupoids. For more detail, see [10, 23].

A groupoid $\mathcal{G}$ is a small category with inverses. We denote the set of identity morphisms of $\mathcal{G}$ by $\mathcal{G}^{(0)}$, and call it the unit space of $\mathcal{G}$. So $\mathcal{G}^{(0)}=\left\{\gamma \gamma^{-1}: \gamma \in \mathcal{G}\right\}$. For $\gamma \in \mathcal{G}$, we write $r(\gamma):=\gamma \gamma^{-1}$ and $s(\gamma):=\gamma^{-1} \gamma$. So $r, s: \mathcal{G} \rightarrow \mathcal{G}^{(0)}$ satisfy $r(\gamma) \gamma=\gamma=\gamma s(\gamma)$ for all $\gamma \in \mathcal{G}$. A pair $(\alpha, \beta) \in \mathcal{G} \times \mathcal{G}$ is then composable if and only if $s(\alpha)=r(\beta)$. We write $\mathcal{G}^{(2)}$ for the set of all composable pairs.

For $U, V \subseteq \mathcal{G}$, we write

$$
U V=\{\alpha \beta \mid \alpha \in U, \beta \in V \text { and } r(\beta)=s(\alpha)\} .
$$

Given units $u, v \in \mathcal{G}^{(0)}$, we write, as usual (e.g. [25]), $\mathcal{G}_{u}$ for $s^{-1}(u)$ and $\mathcal{G}^{v}$ for $r^{-1}(v)$. We then write $\mathcal{G}_{u}^{v}$ for $\mathcal{G}^{v} \cap \mathcal{G}_{u}$. The isotropy group at the unit $u \in \mathcal{G}^{(0)}$ is then the group $\mathcal{G}_{u}^{u}$. We say $u$ has trivial isotropy if $\mathcal{G}_{u}^{u}=\{u\}$. The isotropy subgroupoid of $\mathcal{G}$ is $\operatorname{Iso}(\mathcal{G}):=\bigcup_{u \in \mathcal{G}^{0}} \mathcal{G}_{u}^{u}$. 
We say that $\mathcal{G}$ is a Hausdorff groupoid if it is endowed with a Hausdorff topology under which the range, source and inverse maps are continuous, and the composition map is continuous with respect to the subspace topology on $\mathcal{G}^{(2)} \subseteq \mathcal{G} \times \mathcal{G}$. This implies, in particular, that both $\mathcal{G}^{(0)}$ and $\operatorname{Iso}(\mathcal{G})$ are closed in $\mathcal{G}$. We say $\mathcal{G}$ is étale if $r$ and $s$ are both local homeomorphisms. In this case, the topology on $\mathcal{G}$ has a basis of local bisections 1 : subsets $U \subseteq \mathcal{G}$ such that $r_{\left.\right|_{U}}$ and $s_{\left.\right|_{U}}$ are homeomorphisms onto open subsets of $\mathcal{G}^{(0)}$. This guarantees that $\mathcal{G}^{(0)}$ is clopen in $\mathcal{G}$. We say that $\mathcal{G}$ is ample if it is étale and $\mathcal{G}^{(0)}$ has a basis of compact open sets. If $\mathcal{G}$ is an ample Hausdorff groupoid, then $\mathcal{G}$ admits a basis of compact open local bisections; since $\mathcal{G}$ is Hausdorff, these compact open sets are clopen. The composition map in an ample Hausdorff groupoid is an open map. In this paper we deal only with Hausdorff groupoids. We will work frequently with topologically principal groupoids in which the set of units with trivial isotropy is dense in $\mathcal{G}^{(0)}$.

Recall (see e.g. [10, 18, 23, 27] for more details) that an inverse semigroup is a semigroup $S$ such that for each $s \in S$ there exists a unique element $s^{*} \in S$ satisfying $s s^{*} s=s$ and $s^{*} s s^{*}=s^{*}$. We denote by $E(S)$ the set of idempotents of $S$, which is automatically a commutative semigroup. If $S$ has an element 0 such that $0 s=0=s 0$ for all $s \in S$, then we call $S$ an inverse semigroup with 0 . There is a natural order on $E(S)$ given by $e \leq f$ if and only if $e f=e$, and this order extends to a partial order on $S$ given by $s \leq t$ if $s=$ et for some idempotent $e$ (in which case we may always take $e=s s^{*}$ ). Given an ample Hausdorff groupoid $\mathcal{G}$, the collection $S_{\mathcal{G}}$ of compact open local bisections of $\mathcal{G}$ forms an inverse semigroup 2 with 0 under the multiplication given by (2.1), with $U^{*}=U^{-1}=\left\{\gamma^{-1}: \gamma \in U\right\}$. We then have $E\left(S_{\mathcal{G}}\right):=\left\{U \in S_{\mathcal{G}}: U \subseteq \mathcal{G}^{(0)}\right\}$, and the product in $E\left(S_{\mathcal{G}}\right)$ agrees with the intersection operation on subsets of $\mathcal{G}^{(0)}$. The zero element of $S_{\mathcal{G}}$ is the empty local bisection $\emptyset$.

If $S$ is an inverse semigroup with 0 , and $\Gamma$ is a discrete group, then a $\Gamma$-grading of $S$ is a map $c: S \backslash\{0\} \rightarrow \Gamma$ such that whenever $s, t \in S$ satisfy $s t \neq 0$, we have $c(s t)=c(s) c(t)$.

2.2. Graded Steinberg algebras. Let $\mathcal{G}$ be a Hausdorff ample groupoid and let $R$ be a commutative ring with 1 . We write $A_{R}(\mathcal{G})$ for the space of all locally constant functions $f: \mathcal{G} \rightarrow R$ with compact support. This becomes an $R$-algebra under the convolution product

$$
f * g(\gamma)=\sum_{r(\alpha)=r(\gamma)} f(\alpha) g\left(\alpha^{-1} \gamma\right)=\sum_{\alpha \beta=\gamma} f(\alpha) g(\beta)
$$

and pointwise addition and $R$-action. For any involution on $R$ (possibly the trivial one) the algebra $A_{R}(\mathcal{G})$ becomes a ${ }^{*}$-algebra with $f^{*}(\gamma)=f\left(\gamma^{-1}\right)^{*}$. For $f \in A_{R}(\mathcal{G})$, we write $\operatorname{supp}(f)$ for the support $\{\gamma \in \mathcal{G}: f(\gamma) \neq 0\}$.

For the majority of this paper, we will be interested in the situation where $R$ is in fact an integral domain. We will write $R^{\times}$for the group of units of $R$.

Note that $A_{R}(\mathcal{G})=\operatorname{span}_{R}\left\{1_{U} \mid U \in S_{\mathcal{G}}\right\}$. Specifically, given $f \in A_{R}(\mathcal{G})$, the sets $f^{-1}(r)$, indexed by $r \in R$, are compact open sets, so each admit a finite cover by elements of $S_{\mathcal{G}}$. Since $U \backslash V \in S_{\mathcal{G}}$ whenever $U, V \in S_{\mathcal{G}}$, we can find, for each $r$ such that $f^{-1}(r) \neq \emptyset$, a finite

\footnotetext{
${ }^{1}$ In much of the groupoid literature, these are simply called bisections, or sometimes slices.

${ }^{2}$ Indeed, since $\mathcal{G}$ is Hausdorff, $S_{\mathcal{G}}$ is a Boolean inverse meet-semigroup (see, for example, 31]) with meet given by intersection. But since the additional Boolean meet-semigroup structure comes from the concrete realisation of $S_{\mathcal{G}}$ as a collection of subsets of $\mathcal{G}$, we will not introduce the formal axiomatisation of a Boolean inverse meet-semigroup here, but work concretely with the usual set operations when manipulating elements of $S_{\mathcal{G}}$.
} 
set $F_{r} \subseteq S_{\mathcal{G}}$ of mutually disjoint compact open local bisections with $f^{-1}(r)=\bigsqcup_{U \in F_{r}} U$. We then have $f=\sum_{f^{-1}(r) \neq \emptyset} \sum_{U \in F_{r}} r \cdot 1_{U}$. That is, every element of $A_{R}(\mathcal{G})$ can be written as an $R$-linear combination of finitely many indicator functions of mutually disjoint compact open local bisections. We will use this fact frequently, and, in this context, it will be useful to recall from [27, Proposition 4.5] that for $U, V \in S_{\mathcal{G}}$,

$$
1_{U} * 1_{V}=1_{U V} \quad \text { and } \quad 1_{U}^{*}=1_{U^{-1}} .
$$

Let $\Gamma$ be a discrete group, and $c$ a continuous homomorphism from $\mathcal{G}$ to $\Gamma$ (that is, $c: \mathcal{G} \rightarrow \Gamma$ is a continuous groupoid cocycle). By [9, Lemma 3.1] there is a $\Gamma$-grading of $A_{R}(\mathcal{G})$ such that

$$
A_{R}(\mathcal{G})_{g}=\left\{f \in A_{R}(\mathcal{G}): \operatorname{supp}(f) \subseteq c^{-1}(g)\right\} \quad \text { for all } g \in \Gamma .
$$

We say that a local bisection $U$ is homogeneous if $c(U)$ is a singleton. The collection of all homogeneous compact open local bisections of $\mathcal{G}$ is a $\Gamma$-graded ${ }^{*}$-closed subsemigroup of $S_{\mathcal{G}}$, denoted by $S_{\mathcal{G}, \star}$, under the grading $\bar{c}(U)=g$ if $U \subseteq c^{-1}(g)$. Each homogeneous piece $A_{R}(\mathcal{G})_{g}$ of the Steinberg algebra is then precisely the $R$-linear span of indicator functions of elements of $\bar{c}^{-1}(g)$. As above, each element of $A_{R}(\mathcal{G})_{g}$ can be written as an $R$-linear combination of indicator functions of finitely many mutually disjoint such homogeneous local bisections.

Since we can regard $\mathcal{G}^{(0)}$ as a subgroupoid of $\mathcal{G}$, it makes sense to talk about the Steinberg algebra $A_{R}\left(\mathcal{G}^{(0)}\right)$, which is just the commutative algebra of locally constant compactly supported functions from $\mathcal{G}^{(0)}$ to $R$ under pointwise operations. Since $\mathcal{G}^{(0)}$ is clopen, there is an embedding $\iota: A_{R}\left(\mathcal{G}^{(0)}\right) \rightarrow A_{R}(\mathcal{G})$ such that $\left.\iota(f)\right|_{\mathcal{G}^{(0)}}=f$ and $\left.\iota(f)\right|_{\mathcal{G} \backslash \mathcal{G}^{(0)}}=0$. We use this embedding to regard $A_{R}\left(\mathcal{G}^{(0)}\right)$ as a commutative subalgebra of $A_{R}(\mathcal{G})$. This $A_{R}\left(\mathcal{G}^{(0)}\right)$ contains local units for $A_{R}(\mathcal{G})$ : it contains a set $\mathcal{E}$ of mutually commuting idempotents such that for every finite subset $X$ of $A_{R}(\mathcal{G})$, there exists $e \in \mathcal{E}$ such that $X \subseteq e A_{R}(\mathcal{G}) e$. Specifically, $\mathcal{E}=\left\{1_{K}: K \subseteq \mathcal{G}^{(0)}\right.$ is compact and open $\}$ has the desired property because if $f \in A_{R}(\mathcal{G})$, then $K:=s(\operatorname{supp}(f)) \cup r(\operatorname{supp}(f)) \subseteq \mathcal{G}^{(0)}$ is compact and open, and $1_{K} * f=f=f * 1_{K}$.

To keep our notation uncluttered, we shall write $D_{\mathcal{G}}$, or just $D$ when the groupoid is clear, for $A_{R}\left(\mathcal{G}^{(0)}\right) \subseteq A_{R}(\mathcal{G})$ throughout this note. Observe that when $R$ is an integral domain, the set $E(D)$ of idempotent elements of $D$ is $\left\{1_{K}: K \subseteq \mathcal{G}^{(0)}\right.$ is compact and open $\}$.

We will need the following general result in the proof of our main theorem to show that a homomorphism $\phi: A_{R}(\mathcal{G}) \rightarrow A_{R}(\mathcal{H})$ of Steinberg algebras that carries $D_{\mathcal{G}}$ into the relative commutant of $D_{\mathcal{H}}$ in fact carries $D_{\mathcal{G}}$ onto $D_{\mathcal{H}}$.

Lemma 2.1. Let $\mathcal{G}$ be a topologically principal ample Hausdorff groupoid and $R$ be a commutative integral domain with 1 . Then, $D_{\mathcal{G}}$ is a maximal abelian subring of $A_{R}(\mathcal{G})$.

Proof. Certainly $D_{\mathcal{G}}$ is an abelian subring of $A_{R}(\mathcal{G})$, so we just have to show that it is a maximal abelian subring. For this purpose, suppose that $f \in A_{R}(\mathcal{G}) \backslash D_{\mathcal{G}}$; we must find $a \in D_{\mathcal{G}}$ such that $f a \neq a f$. Fix $\alpha \in \operatorname{supp}(f) \backslash \mathcal{G}^{(0)}$. Because $\operatorname{supp}(f) \subseteq \mathcal{G}$ is open and $\mathcal{G}$ is topologically principal, we may assume that the isotropy at $s(\alpha)$ is trivial; therefore, $s(\alpha) \neq r(\alpha)$. So we can choose disjoint compact open neighbourhoods $V, W \subseteq \mathcal{G}^{(0)}$ of $r(\alpha)$ and $s(\alpha)$, respectively. We now have $\left(1_{V} f 1_{W}\right)(\alpha)=f(\alpha) \neq 0$ whereas $\left(1_{V} 1_{W} f\right)(\alpha)=0$. In particular, $f 1_{W} \neq 1_{W} f$. 


\section{Reconstructing the GROUPoid}

In this section we consider a commutative integral domain $R$ with 1 , and groupoids $\mathcal{G}$ endowed with a continuous cocycle $c$ whose kernel is topologically principal. We show how to reconstruct $(\mathcal{G}, c)$ from the pair $\left(A_{R}(\mathcal{G}), D\right)$, regarded as a graded ring with distinguished abelian subring. Our goal is the following result, which we prove at the end of the section. Throughout $\Gamma$ is a group and $e$ is its neutral element.

Theorem 3.1. Let $\mathcal{G}$ and $\mathcal{H}$ be ample Hausdorff groupoids, let $R$ be a commutative integral domain with 1 , let $c: \mathcal{G} \rightarrow \Gamma$ and $d: \mathcal{H} \rightarrow \Gamma$ be gradings by a discrete group, and suppose that $c^{-1}(e)$ and $d^{-1}(e)$ are topologically principal. Let $D_{\mathcal{G}} \subseteq A_{R}(\mathcal{G})$ and $D_{\mathcal{H}} \subseteq A_{R}(\mathcal{H})$ be the abelian subalgebras consisting of functions supported on $\mathcal{G}^{(0)}$ and $\mathcal{H}^{(0)}$. Then there is a graded isomorphism $\rho: A_{R}(\mathcal{G}) \rightarrow A_{R}(\mathcal{H})$ such that $\rho\left(D_{\mathcal{G}}\right) \subseteq D_{\mathcal{H}}$ if and only if there is an isomorphism $\bar{\rho}: \mathcal{G} \rightarrow \mathcal{H}$ such that $d \circ \bar{\rho}=c$.

Remark 3.2. In Theorem 3.1, we are regarding $A_{R}(\mathcal{G})$ as a ring. In particular, even if $R=\mathbb{C}$, so that $A_{R}(\mathcal{G})$ and $A_{R}(\mathcal{H})$ have natural *-algebra structures, the existence of a diagonal-preserving graded ring isomorphism $A_{R}(\mathcal{G}) \rightarrow A_{R}(\mathcal{H})$ is sufficient to guarantee isomorphism of the groupoids $\mathcal{G}$ and $\mathcal{H}$. En passant we observe that it then follows that $A_{R}(\mathcal{G})$ and $A_{R}(\mathcal{H})$ are in fact isomorphic as ${ }^{*}$-algebras.

3.1. The normaliser of $D_{\mathcal{G}}$. The first step in proving Theorem 3.1 is to define and study what we call the normalisers of $D_{\mathcal{G}}$. As discussed earlier, we often write $D$ instead of $D_{\mathcal{G}}$ for the subalgebra $A_{R}\left(\mathcal{G}^{(0)}\right)$ of $A_{R}(\mathcal{G})$. Our approach is based on Kumjian's work [14] on $C^{*}$-diagonals, and Renault's later work on Cartan subalgebras of $C^{*}$-algebras [26]. It is also related to Brown, Clark and an Huef's treatment of Leavitt path algebras [6] which, in turn, is based on Brownlowe, Carlsen and Whittaker's work on graph $C^{*}$-algebras [7]; but we must make adjustments for the lack of a *-algebra structure here and to exploit the presence of a grading.

Let $\mathcal{G}$ be an ample Hausdorff groupoid, and let $c: \mathcal{G} \rightarrow \Gamma$ be a grading of $\mathcal{G}$ by a discrete group. Note that we have $D \subseteq A_{R}(\mathcal{G})_{e}$, the trivially-graded homogeneous subalgebra of $A_{R}(\mathcal{G})$ since $c(u)=c\left(u^{2}\right)=c(u)^{2}$ for each $u \in \mathcal{G}^{(0)}$.

We shall define what we call the graded normalisers of $D$, and show that they comprise an inverse semigroup. Later we will establish that an appropriate quotient of this inverse semigroup acts on the Stone spectrum of $D$ by partial homeomorphisms, and prove that $\mathcal{G}$ is isomorphic to the groupoid of germs for this action. We are grateful to the referee for suggesting a number of improvements in this section.

Definition 3.3. Let $A$ be a ring. For $n \in A$, let $V(n):=\{m \in A: m n m=m$ and $n m n=$ $n\}$, the set of generalised inverses for $n$ in $A$. Note that $m n$ and $n m$ are idempotents for all $m \in V(n)$. Let $D$ be a commutative subring of $A$ that has local units for $A$ (as described on page 4. We say that $n \in A$ is a normaliser of $D$ if there exists $m \in V(n)$ such that

(N) $m D n \cup n D m \subseteq D$.

In the grading setting, if $A=\bigoplus_{g \in \Gamma} A_{g}$ is a grading of $A$ by a discrete group $\Gamma$, we say that a normaliser $n$ of $D$ is homogeneous if $n \in c^{-1}(g)$ for some $g \in \Gamma$.

Notation 3.4. If $A$ is a ring and $D \subseteq A$ is a commutative subring containing local units for $A$, then we write $N_{A}(D)$, or just $N(D)$ for the collection of all normalisers of $D$ in 
$A$. If $A$ is $\Gamma$-graded, we write $N_{\star}(D)$ for the collection of homogeneous normalisers of $D$, and for $g \in \Gamma$, we write $N_{g}(D)$ for $N_{\star}(G) \cap A_{g}$; we call the elements of $N_{g}(D)$ the $g$-homogeneous normalisers of $D$.

Here, we are interested in the situation where $A=A_{R}(\mathcal{G})$ is the Steinberg algebra of an ample Hausdorff groupoid, $D$ is the diagonal subalgebra of locally constant $R$-valued functions on $\mathcal{G}^{(0)}$, and the grading is given by a cocycle $c: \mathcal{G} \rightarrow \Gamma$. Observe that then $D \subseteq A_{R}(\mathcal{G})_{e}$.

Our first proposition, which is the linchpin of the paper, characterises the homogeneous normalisers of $D$ when $c^{-1}(e)$ is topologically principal. We will use this in Corollary 3.7] to show that $N_{\star}(D)$ is an inverse semigroup. As suggested by the referee, we could probably deduce this from the general theory of inverse semigroups by showing that $N_{\star}(D)$ is a regular semigroup with commuting idempotents; but it will be important later to have the explicit description of elements of $N_{\star}(D)$ given in the following proposition. Recall that $R^{\times}$denotes the group of units of a ring $R$.

Proposition 3.5. Let $\mathcal{G}$ be an ample Hausdorff groupoid, and let $c: \mathcal{G} \rightarrow \Gamma$ be a grading of $\mathcal{G}$ by a discrete group. Let $R$ be a commutative ring with 1 .

(1) Let $U \subseteq c^{-1}(g)$ be a compact open local bisection. Suppose that $F$ is a finite collection of mutually disjoint compact open subsets $V$ of $U$ such that $U=\bigsqcup_{V \in F} V$. Fix units $a_{V} \in R^{\times}$for $V \in F$ and let

$$
n=\sum_{V \in F} a_{V} 1_{V} \quad \text { and } \quad m=\sum_{V \in F} a_{V}^{-1} 1_{V^{-1}}
$$

Then $n \in N(D)$, with $m \in V(n)$ satisfying $(N)$.

(2) Suppose that $R$ is an integral domain and $c^{-1}(e)$ is topologically principal. Suppose that $n \in N_{g}(D)$, and that $m \in V(n)$ satisfies $(N)$. Then there exist a compact open local bisection $U \subseteq c^{-1}(g)$, a decomposition $U=\bigsqcup_{V \in F} V$ of $U$ into finitely many mutually disjoint compact open subsets, and units $a_{V} \in R^{\times}$indexed by $V \in F$ such that

$$
n=\sum_{V \in F} a_{V} 1_{V} \quad \text { and } \quad m=\sum_{V \in F} a_{V}^{-1} 1_{V^{-1}}
$$

We have $n m=1_{r(U)}$ and $m n=1_{s(U)}$.

Proof. (1) Since $D=\operatorname{span}_{R}\left\{1_{K}: K \subseteq \mathcal{G}^{(0)}\right.$ is compact open $\}$, to see that $m$ satisfies (N) it suffices to show that each $n 1_{K} m$ and each $m 1_{K} n$ is contained in $D$. For a compact open $K \subseteq \mathcal{G}^{(0)}$,

$$
n 1_{K} m=\sum_{V, W \in F} a_{V} a_{W}^{-1} 1_{V} 1_{K} 1_{W^{-1}}=\sum_{V, W \in F} a_{V} a_{W}^{-1} 1_{V K} 1_{(W K)^{-1}}
$$

Since $s(V) \cap s(W)=\emptyset$ for distinct $V, W \in F$, it follows that $1_{V K} 1_{(W K)^{-1}}=0$ unless $V=W$. So

$$
n 1_{K} m=\sum_{V \in F} a_{V} a_{V}^{-1} 1_{V K(V K)^{-1}}=\sum_{V \in F} 1_{r(V K)}=1_{r(U K)}
$$

since the sets $V$ are mutually disjoint and cover $U$. Similarly, $m 1_{K} n=1_{s(K U)}$. This establishes $(\mathrm{N})$.

Now, we show that $m \in V(n)$. Applying the two identities just derived with $K=$ $s(U) \cup r(U)$ gives $n m=1_{r(U)}$ and $m n=1_{s(U)}$. Hence $1_{V} m n=n m 1_{V}=1_{V}$ for each 
$V \in F$. So

$$
n m n=\sum_{V \in F} a_{V} 1_{V} 1_{s(U)}=\sum_{V \in F} a_{V} 1_{V s(U)}=\sum_{V \in F} a_{V} 1_{V}=n,
$$

and similarly $m n m=m$.

(22) Let $U_{n}:=\operatorname{supp}(n) \subseteq c^{-1}(g)$. Since $R$ is an integral domain, $\operatorname{supp}(m) \operatorname{supp}(n)=$ $\operatorname{supp}(m n) \subseteq \mathcal{G}^{(0)} \subseteq \mathcal{G}_{e}$, and so $U_{m}:=\operatorname{supp}(m)$ is a subset of $c^{-1}\left(g^{-1}\right)$. The sets $U_{n}$ and $U_{m}$ are compact open sets because $n, m \in A_{R}(\mathcal{G})$. We will show that $U_{n}$ is a local bisection and that $U_{m}=U_{n}^{-1}$. First observe that $m n$ is an idempotent in $D=A_{R}\left(\mathcal{G}^{(0)}\right)$. Since $R$ is an integral domain, it has no nontrivial idempotents, and since multiplication in $D$ is pointwise, we conclude that $m n=1_{K}$ for some compact open set $K$. We claim that $K=s\left(U_{n}\right)$. For this, first suppose that $u \in K$. Then

$$
0 \neq m n(u)=\sum_{\alpha \beta=u} m(\alpha) n(\beta)=\sum_{s(\alpha)=u} m\left(\alpha^{-1}\right) n(\alpha) .
$$

So $n(\alpha) \neq 0$ for some $\alpha \in \mathcal{G}_{u}$, giving $u \in s(\operatorname{supp}(n))=s\left(U_{n}\right)$. Hence $K \subseteq s\left(U_{n}\right)$. For the reverse inclusion, suppose that $u \in s\left(U_{n}\right)$; that is, there exists $\alpha \in U_{n}$ satisfying $u=s(\alpha)$. Then

$$
0 \neq n(\alpha)=n m n(\alpha)=\sum_{\beta \gamma=\alpha} n(\beta) m n(\gamma)=\sum_{\beta \gamma=\alpha} n(\beta) 1_{K}(\gamma)
$$

Since $K \subseteq \mathcal{G}^{(0)}$, the rightmost sum in the preceding equation collapses to $n(\alpha) 1_{K}(u)$. Moreover, as it is nonzero, we conclude that $u \in K$, and then, $m n=1_{s\left(U_{n}\right)}$. The same argument applied to the homogeneous normaliser $m$ shows that $n m=1_{s\left(U_{m}\right)}$. Similarly, $n m=1_{r\left(U_{n}\right)}$ and $m n=1_{r\left(U_{m}\right)}$. Therefore, $s\left(U_{n}\right)=r\left(U_{m}\right)$ and $s\left(U_{m}\right)=r\left(U_{n}\right)$.

Now, fixing $u \in s\left(U_{n}\right)$, we show that the set $U_{n} u U_{m}$ consists of a single element of $\mathcal{G}^{(0)}$. Since

$$
0 \neq 1_{s\left(U_{n}\right)}(u)=m n(u)=\sum_{\alpha \beta=u} m(\alpha) n(\beta)=\sum_{s(\alpha)=u} m\left(\alpha^{-1}\right) n(\alpha),
$$

there exists $\alpha_{0} \in U_{n} u$ such that $\alpha_{0}^{-1} \in U_{m}$; we must show that $U_{n} u U_{m}=\left\{r\left(\alpha_{0}\right)\right\}$. Since inversion in $\mathcal{G}$ is a homeomorphism, and since $n, m$ are locally constant, we can choose an open local bisection $V_{\alpha_{0}}^{0}$ such that $\alpha_{0} \in V_{\alpha_{0}}^{0} \subseteq U_{n}$, such that $\left(V_{\alpha_{0}}^{0}\right)^{-1} \subseteq U_{m}$, and such that $n$ is constant on $V_{\alpha_{0}}^{0}$ and $m$ is constant on $\left(V_{\alpha_{0}}^{0}\right)^{-1}$. Let $W_{\alpha_{0}^{-1}}^{0}:=\left(V_{\alpha_{0}}^{0}\right)^{-1}$. The sets $U_{n} u \backslash\left\{\alpha_{0}\right\}$ and $u U_{m} \backslash\left\{\alpha_{0}^{-1}\right\}$ are finite and discrete because $r$ and $s$ are local homeomorphisms and $U_{n}$ and $U_{m}$ are compact. For each $\alpha \in U_{n} u \backslash\left\{\alpha_{0}\right\}$, choose an open local bisection $V_{\alpha}^{0}$ with $\alpha \in V_{\alpha}^{0} \subseteq U_{n}$ such that $n$ is constant on $V_{\alpha}^{0}$; and for each $\beta \in u U_{m} \backslash\left\{\alpha_{0}^{-1}\right\}$ choose an open local bisection $\beta \in W_{\beta}^{0} \subseteq U_{m}$ with $m$ constant on $W_{\beta}^{0}$. The set $Y:=\left(\bigcap_{\alpha \in U_{n}} s\left(V_{\alpha}^{0}\right)\right) \cap\left(\bigcap_{\beta \in u U_{m}} r\left(W_{\beta}^{0}\right)\right)$ is open. For each $\alpha \in U_{n} u$, put $V_{\alpha}:=V_{\alpha}^{0} Y$, and for each $\beta \in u U_{m}$, let $W_{\beta}:=Y W_{\beta}^{0}$. Then:

- $W_{\alpha_{0}^{-1}}=V_{\alpha_{0}}^{-1}$

- each $V_{\alpha}$ is an open local bisection containing $\alpha$, and each $W_{\beta}$ is an open local bisection containing $\beta$;

- $n$ is constant on each $V_{\alpha}$ and $m$ is constant on each $W_{\beta}$; and

- $s\left(V_{\alpha}\right)=Y=r\left(W_{\beta}\right)$ for all $\alpha, \beta$.

As $U_{n} \subseteq c^{-1}(g)$ and $U_{m} \subseteq c^{-1}\left(g^{-1}\right)$, we have $V_{\alpha} W_{\beta} \subseteq c^{-1}(e)$ for all $\alpha, \beta$. Because $Y$ is open, and since $c^{-1}(e)$ is topologically principal, we can find $y \in Y$ such that 
$\mathcal{G}_{y}^{y} \cap c^{-1}(e)=\{y\}$. It follows that if $\mu, \nu \in U_{n} y$ are distinct then $r(\mu) \neq r(\nu)$ (because otherwise $\mu^{-1} \nu$ would belong to $\mathcal{G}_{y}^{y} \cap c^{-1}(e) \backslash\{y\}$ ), and similarly, if $\mu, \nu \in y U_{n}$ are distinct then $s(\mu) \neq s(\nu)$. So we can choose a compact open neighbourhood $V_{\eta}^{\prime}$ of each $\eta \in U_{n} y$ and a compact open neighbourhood $W_{\zeta}^{\prime}$ of each $\zeta \in y U_{m}$ such that the $r\left(V_{\eta}^{\prime}\right)$ are mutually disjoint and the $s\left(W_{\zeta}^{\prime}\right)$ are mutually disjoint; and we can assume that $V_{\eta}^{\prime} \subseteq V_{\alpha}$ if $\eta \in V_{\alpha}$, and similarly for the $W_{\zeta}^{\prime}$. Let $X:=\left(\bigcap_{\eta \in U_{n} y} s\left(V_{\eta}^{\prime}\right)\right) \cap\left(\bigcap_{\zeta \in y U_{m}} r\left(W_{\zeta}^{\prime}\right)\right)$. Since $1_{r\left(V_{\eta}^{\prime}\right)} 1_{r\left(V_{\eta^{\prime}}^{\prime}\right)}=0$ for distinct $\eta, \eta^{\prime} \in U_{n} y$, and $1_{s\left(W_{\zeta^{\prime}}^{\prime}\right)} 1_{s\left(W_{\zeta}^{\prime}\right)}=0$ for distinct $\zeta, \zeta^{\prime} \in y U_{m}$, we have

$$
\left(1_{r\left(V_{\eta}^{\prime}\right)} n 1_{X} m 1_{s\left(W_{\zeta}^{\prime}\right)}\right)(\eta \zeta)=n(\eta) m(\zeta)
$$

for each $\eta \in U_{n} y$ and $\zeta \in y U_{m}$. Now we suppose, that $U_{n} u U_{m}$ is not a singleton, and derive a contradiction. Either there exists $\alpha_{1} \in U_{n} u \backslash\left\{\alpha_{0}\right\}$ or there is $\beta_{1} \in u U_{m} \backslash\left\{\alpha_{0}^{-1}\right\}$. We consider the former case; the latter is similar. Let $\eta_{0}$ and $\eta_{1}$ be the unique elements of $V_{\alpha_{0}} y$ and $V_{\alpha_{1}} y$. Since $W_{\alpha_{0}^{-1}}=V_{\alpha_{0}}^{-1}$, the unique element of $y W_{\alpha_{0}^{-1}}$ is $\zeta_{0}:=\eta_{0}^{-1}$. As $m, n$ satisfy $(\mathrm{N})$, we have $n 1_{X} m \in D$ and therefore $1_{r\left(V_{\eta_{1}}^{\prime}\right)} n 1_{X} m 1_{s\left(W_{\zeta_{0}}^{\prime}\right)} \in D$. But (3.1) and that $n$ is constant on $V_{\alpha_{1}}$ and $m$ is constant on $W_{\alpha_{0}^{-1}}$ gives

$$
\left(1_{r\left(V_{\eta_{1}}^{\prime}\right)} n 1_{X} m 1_{s\left(W_{\zeta_{0}}^{\prime}\right)}\right)\left(\eta_{1} \zeta_{0}\right)=n\left(\eta_{1}\right) m\left(\zeta_{0}\right)=n\left(\alpha_{1}\right) m\left(\alpha_{0}^{-1}\right)
$$

We have $n\left(\alpha_{1}\right) \neq 0$ and $m\left(\alpha_{0}^{-1}\right) \neq 0$ because $\alpha_{1} \in U_{n}=\operatorname{supp}(n)$ and $\alpha_{0}^{-1} \in U_{m}=$ $\operatorname{supp}(m)$; since $R$ is an integral domain, we deduce that $\left(1_{r\left(V_{\eta_{1}}^{\prime}\right)} n 1_{X} m 1_{s\left(W_{\zeta_{0}}^{\prime}\right)}\right)\left(\eta_{1} \zeta_{0}\right) \neq 0$. Since $\zeta_{0}=\eta_{0}^{-1} \neq \eta_{1}^{-1}$, we have $\eta_{1} \zeta_{0} \notin \mathcal{G}^{(0)}$, which contradicts $1_{r\left(V_{\eta_{1}}^{\prime}\right)} n 1_{X} m 1_{s\left(W_{\zeta_{0}}^{\prime}\right)} \in D$.

We have now established that $U_{n}$ is a local bisection, and symmetry shows that $U_{m}$ is a local bisection. We also showed at the beginning of the preceding paragraph that if $u \in s\left(U_{n}\right)$ then there exists $\alpha \in U_{n} u$ such that $\alpha^{-1} \in U_{m}$. Since we now also know that $U_{n} u$ is a singleton, we deduce that $U_{n}^{-1} \subseteq U_{m}$; and symmetry implies that in fact $U_{m}=U_{n}^{-1}$. Since $n$ and $m$ are locally constant, we can express $U_{n}=\bigsqcup_{V \in F} V$ where the $V$ are mutually disjoint compact open sets such that $n$ is constant (and nonzero) on $V$ and $m$ is constant on $V^{-1}$ for each $V \in F$; say $n \equiv r_{V}$ on $V$ and $m \equiv r_{V}^{\prime}$ on $V^{-1}$. So $n=\sum_{V \in F} r_{V} 1_{V}$ and $m=\sum_{V \in F} r_{V}^{\prime} 1_{V^{-1}}$. We just have to show that each $r_{V} r_{V}^{\prime}=1$. Since $U$ is a local bisection, the sets $r(V)$ are mutually disjoint, and so for $w \in r(U)$ there is a unique $V$ such that $w \in r(V)$. We then have

$$
n m(w)=\sum_{r(\alpha)=w} n(\alpha) m\left(\alpha^{-1}\right)=r_{V} r_{V^{-1}}^{\prime}
$$

We have $w \in r\left(U_{n}\right)$ and we proved that $n m=1_{r\left(U_{n}\right)}$. Hence $r_{V} r_{V^{-1}}^{\prime}=1$ as required.

Remark 3.6. (1) If $U \subseteq c^{-1}(g)$ is a compact open local bisection, then $1_{U} \in N_{g}(D)$, with $m=1_{U^{-1}} \in V\left(1_{U}\right)$ satisfying $(\mathrm{N})$. Hence $A_{R}(\mathcal{G})=\operatorname{span}_{R}\left(N_{\star}(D)\right)$.

(2) Since $D$ has local units for $A_{R}(G)$ (see page 4), if $n \in N(D)$, with $m \in V(n)$ satisfying $(\mathrm{N})$, then $n m, m n \in D$.

3.2. The inverse semigroup $N_{\star}(D)$. We now show that $N_{\star}(D)$ is an inverse semigroup carrying a $\Gamma$-grading. In particular, we will show that a quotient $N_{\star}(D) / \sim$ of this inverse semigroup acts on the Stone spectrum $\widehat{D}$, so that we can construct from it a $\Gamma$-graded groupoid of germs $\left(N_{\star}(D) / \sim\right) \times_{\varphi} \widehat{D}$. 
We write $C_{\mathrm{lc}}\left(\mathcal{G}, R^{\times} \cup\{0\}\right)$ for the set $\left\{f \in A_{R}(\mathcal{G}): f(\mathcal{G}) \subseteq R^{\times} \cup\{0\}\right\}$ of functions in $A_{R}(\mathcal{G})$ whose nonzero values are units. We define ${ }^{*}: C_{\mathrm{lc}}\left(\mathcal{G}, R^{\times} \cup\{0\}\right) \rightarrow C_{\mathrm{lc}}\left(\mathcal{G}, R^{\times} \cup\{0\}\right)$ by

$$
f^{*}(\gamma)= \begin{cases}f\left(\gamma^{-1}\right)^{-1} & \text { if } f\left(\gamma^{-1}\right) \neq 0 \\ 0 & \text { otherwise. }\end{cases}
$$

It will be convenient to write $r^{*}:=r^{-1}$ for $r \in R^{\times}$and $0^{*}:=0$. Under this notation, we have $f^{*}(\gamma)=f\left(\gamma^{-1}\right)^{*}$ for all $f \in C_{\mathrm{lc}}\left(\mathcal{G}, R^{\times} \cup\{0\}\right)$ and $\gamma \in \mathcal{G}$.

We define

$$
C_{\mathrm{lc}}\left(\mathcal{G}, R^{\times} \cup\{0\}\right)_{\star}:=\left\{f \in C_{\mathrm{lc}}\left(\mathcal{G}, R^{\times} \cup\{0\}\right): f \text { is homogeneous }\right\},
$$

and for $g \in \Gamma$, we write $C_{\mathrm{lc}}\left(\mathcal{G}, R^{\times} \cup\{0\}\right)_{g}:=C_{\mathrm{lc}}\left(\mathcal{G}, R^{\times} \cup\{0\}\right) \cap A_{R}(\mathcal{G})_{g}$.

We can reinterpret Proposition 3.5 as follows.

Corollary 3.7. Let $\mathcal{G}$ be an ample Hausdorff groupoid, and let $c: \mathcal{G} \rightarrow \Gamma$ be a grading of $\mathcal{G}$ by a discrete group. Suppose that $c^{-1}(e)$ is topologically principal. Let $R$ be a commutative integral domain with 1 . Then

$$
N_{\star}(D)=\left\{f \in C_{\mathrm{lc}}\left(\mathcal{G}, R^{\times} \cup\{0\}\right)_{\star}: \operatorname{supp}(f) \text { is a local bisection }\right\},
$$

and the map $f \mapsto f^{*}$ is an involution on $N_{\star}(D)$ satisfying $f^{*} f=1_{s(\operatorname{supp}(f))}$ and $f f^{*}=$ $1_{r(\operatorname{supp}(f))}$ for $f \in N_{\star}(D)$. Moreover, $N_{\star}(D)$ is an inverse semigroup, and there is a $\Gamma$-grading $\tilde{c}: N_{\star}(D) \backslash\{0\} \rightarrow \Gamma$ defined by $\tilde{c}(f)=g$ if and only if $f \in N_{g}(D)$.

Proof. Proposition 3.5 gives (3.3) holds. If $f \in N_{\star}(D)$, then clearly $f^{*} \in N_{\star}(D)$ and $f^{* *}=f$. Since $R$ is an integral domain, for $f, g \in N_{\star}(D)$, and $\gamma \in \mathcal{G}$, we have $\gamma \in \operatorname{supp}(f g)$ if and only if $\gamma \in \operatorname{supp}(f) \operatorname{supp}(g)$; and in this case, there are unique elements $\alpha \in \operatorname{supp}(f)$ and $\beta \in \operatorname{supp}(g)$ such that $\gamma=\alpha \beta$. If $\gamma \in \operatorname{supp}\left(g^{*}\right) \operatorname{supp}\left(f^{*}\right)$, it follows that

$$
\begin{aligned}
(f g)^{*}(\gamma)=(f g)\left(\gamma^{-1}\right)^{-1} & =\left(f\left(\eta^{-1}\right) g\left(\xi^{-1}\right)\right)^{-1} \\
& =g\left(\xi^{-1}\right)^{-1} f\left(\eta^{-1}\right)^{-1}=g^{*}(\xi) f^{*}(\eta)=\left(g^{*} f^{*}\right)(\gamma),
\end{aligned}
$$

where $\xi \in \operatorname{supp}\left(g^{*}\right)$ and $\eta \in \operatorname{supp}\left(f^{*}\right)$ are the unique elements such that $\gamma=\xi \eta$. On the other hand, if $\gamma \notin \operatorname{supp}\left(g^{*}\right) \operatorname{supp}\left(f^{*}\right)$, then $(f g)^{*}(\gamma)=0=\left(g^{*}\right)\left(f^{*}\right)(\gamma)$. So ${ }^{*}$ is an involution on $N_{\star}(D)$. (We did not need commutativity of $R$ for this computation.)

If $f \in N_{\star}(D)$ and $\gamma \in \mathcal{G}$, then the displayed equation above shows that $\left(f f^{*}\right)(\gamma)=0$ except when $\gamma \in \operatorname{supp}(f) \operatorname{supp}(f)^{-1}=r(\operatorname{supp}(f))$, because $\operatorname{supp}(f)$ is a local bisection. Moreover, if $\gamma=\alpha \alpha^{-1}=r(\alpha)$ for $\alpha \in \operatorname{supp}(f)$, then $\left(f f^{*}\right)(\gamma)=f(\alpha) f\left(\alpha^{-1}\right)^{-1}=1$. Hence $f f^{*}=1_{r(\operatorname{supp}(f))}$. An identical calculation shows that $f^{*} f=1_{s(\operatorname{supp}(f))}$. It follows immediately that $f f^{*} f=f$ and $f^{*} f f^{*}=f^{*}$, so that $f^{*} \in V(f)$. For $K \subseteq \mathcal{G}^{(0)}$ compact open, we have $f 1_{K}=\left.f\right|_{\operatorname{supp}(f) K} \in N_{\star}(D)$, and likewise $1_{K} f \in N_{\star}(D)$; thus, $f 1_{K} f^{*}=$ $\left(f 1_{K}\right)\left(1_{K} f^{*}\right)$ and $f^{*} 1_{K} f=\left(1_{K} f\right)^{*}\left(1_{K} f\right)$ belong to $D$ by the reasoning just applied to $f f^{*}$ and $f^{*} f$. Since $D$ is the $R$-linear span of the $1_{K}$, it follows that $f D f^{*} \cup f^{*} D f \subseteq D$. So we have established that the pair $\left(f, f^{*}\right)$ satisfies $(\mathrm{N})$. This implies that the map $f \mapsto f^{*}$ is an involution on $N_{\star}(D)$ satisfying the desired properties.

To see that $N_{\star}(D)$ is an $\Gamma$-graded inverse semigroup, fix $f_{1}, f_{2} \in N_{\star}(D)$. Each $\gamma \in$ $\operatorname{supp}\left(f_{1} f_{2}\right)$ can be factorised uniquely as $\gamma=\gamma_{1} \gamma_{2}$ with $\gamma_{1} \in \operatorname{supp}\left(f_{1}\right)$ and $\gamma_{2} \in \operatorname{supp}\left(f_{2}\right)$. Since $R^{\times}$is closed under multiplication,

$$
\left(f_{1} f_{2}\right)(\gamma)=f_{1}\left(\gamma_{1}\right) f_{2}\left(\gamma_{2}\right) \in R^{\times} \cdot R^{\times} \in R^{\times} \cup\{0\} .
$$


So $N_{\star}(D)$ is closed under multiplication. The set $N_{\star}(D)$ is clearly closed under ${ }^{*}$, and * is an involution on $N_{\star}(D)$ such that $f f^{*} f=f$ and $f^{*} f f^{*}=f^{*}$ by the preceding paragraph. The idempotents of $N_{\star}(D)$ are of the form $1_{K}$, where $K$ is an open compact subset of $\mathcal{G}^{(0)}$. So they commute, and hence $N_{\star}(D)$ is an inverse semigroup by [18, Theorem 1.3].

Let $S_{\mathcal{G}, \star}$ be the $\Gamma$-graded inverse semigroup of homogeneous compact open local bisections of $\mathcal{G}$, with grading $\bar{c}$ as established in Section 2.2. Since $R$ is an integral domain, if $f_{1}, f_{2} \in N_{\star}(D)$, then $\operatorname{supp}\left(f_{1} f_{2}\right)=\operatorname{supp}\left(f_{1}\right) \operatorname{supp}\left(f_{2}\right)$. Hence $f_{1} f_{2}=0$ if and only if $\operatorname{supp}\left(f_{1}\right) \operatorname{supp}\left(f_{2}\right)=\emptyset$. Since $\tilde{c}(f)=\bar{c}(\operatorname{supp}(f))$ for all $f$, it follows that $\tilde{c}$ is a $\Gamma$ grading.

3.3. From $N_{\star}(D)$ to the inverse semigroup of compact open local bisections. The collection $S_{\mathcal{G}, \star}$ of homogeneous compact open local bisections (including the empty local bisection) of $\mathcal{G}$ forms an inverse semigroup under composition and with involution $U \mapsto U^{-1}$. We now pass from the inverse semigroup $N_{\star}(D)$ described in the preceding section to a quotient $N_{\star}(D) / \sim$, which we prove is isomorphic to $S_{\mathcal{G}, \star}$.

We write $E(D)$ for the boolean ring of idempotent elements of $D$.

Lemma 3.8. Let $\mathcal{G}$ be an ample Hausdorff groupoid, and let $c: \mathcal{G} \rightarrow \Gamma$ be a grading of $\mathcal{G}$ by a discrete group. Suppose that $c^{-1}(e)$ is topologically principal. Let $R$ be a commutative integral domain with 1 , and let $\tilde{c}: N_{\star}(D) \backslash\{0\} \rightarrow \Gamma$ be the $\Gamma$-grading of Corollary 3.7. Then there is an equivalence relation $\sim$ on $N_{\star}(D)$ defined by $f \sim h$ if and only if all of the following three conditions are satisfied:

(1) $\tilde{c}(f)=\tilde{c}(h)$,

(2) $f^{*} p f=h^{*} p h$ for every $p \in E(D)$, and

(3) $f_{p} f^{*}=h p h^{*}$ for every $p \in E(D)$.

Moreover, the set $N_{\star}(D) / \sim$ is an inverse semigroup under the operations $[f][g]=[f g]$ and $[f]^{*}=\left[f^{*}\right]$. The map $q: N_{\star}(D) \rightarrow S_{\mathcal{G}, \star}$ given by $q(f)=\operatorname{supp}(f)$ induces an isomorphism of inverse semigroups $\tilde{q}: N_{\star}(D) / \sim \rightarrow S_{\mathcal{G}, \star}$.

Proof. It suffices to show that $q: N_{\star}(D) \rightarrow S_{\mathcal{G}, \star}$ is a surjective semigroup homomorphism, and that $q(f)=q(h)$ if and only if $f \sim h$.

Since $R$ is an integral domain, we have $\operatorname{supp}(f h)=\operatorname{supp}(f) \operatorname{supp}(h)$ for all $f, h \in$ $N_{\star}(D)$, and we have $\operatorname{supp}\left(f^{*}\right)=\operatorname{supp}(f)^{-1}$ by definition of ${ }^{*}$ on $N_{\star}(D)$. So the map $q$ is a semigroup homomorphism. It is surjective because each homogeneous compact open local bisection $V$ satisfies $V=q\left(1_{V}\right)$.

To see that $q(f)=q(h) \Longleftrightarrow f \sim h$, first suppose that $q(f)=q(h)$. Then $\operatorname{supp}(f)=$ $\operatorname{supp}(h)$. Since $\tilde{c}(f)=g$ if and only if $\operatorname{supp}(f) \subseteq c^{-1}(g)$, it follows that $\tilde{c}(f)=\tilde{c}(h)$. If $p \in D$ is an idempotent, then $p=1_{K}$ for some compact open $K \subseteq \mathcal{G}^{(0)}$. We then have $p f=\left.f\right|_{K \operatorname{supp}(f)}$ and $p h=\left.h\right|_{K \operatorname{supp}(h)}=\left.h\right|_{K \operatorname{supp}(f)}$. Thus, Corollary 3.7 applied to $p f, p h \in N_{\star}(D)$ shows that

$$
\begin{aligned}
f^{*} p f & =(p f)^{*}(p f)=1_{s(K \operatorname{supp}(f))}=1_{s(K q(f))} \\
& =1_{s(K q(h))}=1_{s(K \operatorname{supp}(h))}=(p h)^{*}(p h)=h^{*} p h
\end{aligned}
$$

and similarly $f p f^{*}=h p h^{*}$.

Now suppose $f \sim h$. We must show that $\operatorname{supp}(f)=\operatorname{supp}(h)$. Since $\tilde{c}(f)=\tilde{c}(h)$, we have $\operatorname{supp}(f) \operatorname{supp}(h)^{-1} \subseteq c^{-1}(e)$ which is topologically principal. Putting $p=$ $1_{r(\operatorname{supp}(f)) \cup r(\operatorname{supp}(h))}$ in (2), we see that $f^{*} f=h^{*} h$, and so $s(\operatorname{supp}(f))=s(\operatorname{supp}(h))$. We will show that $\operatorname{supp}(f) \cap \operatorname{supp}(h)$ is dense in $\operatorname{supp}(f)$. Let $U$ be a non-empty open 
subset of $\mathcal{G}$ contained in $\operatorname{supp}(f)$. Since $c^{-1}(e)$ is topologically principal and $r(U)$ is open, there is $\alpha \in U$ such that the isotropy at $r(\alpha)$ is trivial. Let $\beta \in \operatorname{supp}(h)$ be the unique element with $s(\alpha)=s(\beta)$. We suppose that $\beta \neq \alpha$ to derive a contradiction. Since the isotropy at $r(\alpha)$ is trivial, and $\beta \neq \alpha$, we have $r(\beta) \neq r(\alpha)$; thus, there exist disjoint compact open sets $V, W \subseteq \mathcal{G}^{(0)}$ such that $r(\alpha) \in V$ and $r(\beta) \in W$. Let $X=s(V \operatorname{supp}(f)) \cap s(W \operatorname{supp}(h))$. Then $\left(f 1_{X} f^{*}\right)(r(\alpha))=1$ and $\left(h 1_{X} h^{*}\right)(r(\alpha))=0$, contradicting (3). This shows that $\operatorname{supp}(f) \cap \operatorname{supp}(h)$ is dense in $\operatorname{supp}(f)$. Since $\mathcal{G}$ is Hausdorff, the compact sets $\operatorname{supp}(f)$ and $\operatorname{supp}(h)$ are closed, and so $\operatorname{supp}(f) \cap \operatorname{supp}(h)$ is closed. Hence $\operatorname{supp}(f) \cap \operatorname{supp}(h)=\operatorname{supp}(f)$. Similarly $\operatorname{supp}(f) \cap \operatorname{supp}(h)=\operatorname{supp}(h)$ and thus $\operatorname{supp}(f)=\operatorname{supp}(h)$.

Since $R$ has no nontrivial idempotent elements, the Boolean ring $E(D)$ is precisely the set $\left\{1_{K}: K \subseteq \mathcal{G}^{(0)}\right.$ is compact open $\}$, and so corresponds to the Boolean algebra of compact open subsets of $\mathcal{G}^{(0)}$. We write $\widehat{D}$ for the Stone spectrum of $E(D)$ : that is, the space of Boolean-ring homomorphisms $\pi: E(D) \rightarrow\{0,1\}$. By Stone duality, there is a homeomorphism $\varepsilon: \mathcal{G}^{(0)} \rightarrow \widehat{D}$ such that $\varepsilon_{u}(p):=p(u)$ for $p \in E(D)$; the inverse of this map takes a Boolean-ring homomorphism $\pi: E(D) \rightarrow\{0,1\}$ to the unique point in $\left(\bigcap_{\pi\left(1_{K}\right)=1} K\right) \backslash\left(\bigcup_{\pi\left(1_{K}\right)=0} K\right)$.

Recall that there is an action $\theta$ of $S_{\mathcal{G}, \star}$ on $\mathcal{G}^{(0)}$ such that $\operatorname{dom}\left(\theta_{V}\right)=s(V), \operatorname{cod}\left(\theta_{V}\right)=$ $r(V)$ and $\theta_{V}(s(\alpha))=r(\alpha)$ for all $\alpha \in V$.

Lemma 3.9. Let $\mathcal{G}$ be an ample Hausdorff groupoid, let $c: \mathcal{G} \rightarrow \Gamma$ be a grading of $\mathcal{G}$ by a discrete group with $c^{-1}(e)$ topologically principal, let $R$ be a commutative integral domain with 1 , and let $N_{\star}(D) / \sim$ be the inverse semigroup of Lemma 3.8. With $\varepsilon: \mathcal{G}^{(0)} \rightarrow \widehat{D}$ and $\theta: S_{\mathcal{G}, \star} \curvearrowright \mathcal{G}^{(0)}$ as above, and $\tilde{q}: N_{\star}(D) / \sim \rightarrow S_{\mathcal{G}, \star}$ as in Lemma 3.8, we have

$$
\varepsilon_{\theta_{\tilde{q}(f)}(u)}(p)=\varepsilon_{u}\left(f^{*} p f\right) \quad \text { for all } f \in N_{\star}(D) / \sim, u \in s(\operatorname{supp}(f)) \text { and } p \in E(D) .
$$

Proof. Fix $f \in N_{\star}(D), u \in s(\operatorname{supp}(f))$ and $p \in E(D)$. Then $p=1_{K}$ for some compact open $K \subseteq \mathcal{G}^{(0)}$. Let $\alpha \in \operatorname{supp}(f)$ be the unique element with $s(\alpha)=u$. Then

$$
\varepsilon_{\theta_{\tilde{q}(f)}(u)}(p)=p\left(\theta_{\tilde{q}(f)}(u)\right)=1_{K}(r(\alpha)) .
$$

Also,

$$
\begin{aligned}
\varepsilon_{u}\left(f^{*} p f\right)=\left(f^{*} 1_{K} f\right)(u) & =\sum_{\beta \gamma=u}\left(1_{K} f\right)^{*}(\beta) f(\gamma)=\left(1_{K} f\right)^{*}\left(\alpha^{-1}\right) f(\alpha) \\
& =\left(1_{K} f\right)(\alpha)^{*} f(\alpha)=1_{K}(r(\alpha)) f(\alpha)^{*} f(\alpha)=1_{K}(r(\alpha)) .
\end{aligned}
$$

3.4. Groupoids of germs. If $\varphi$ is an action of a countable inverse semigroup $S$ on a locally compact Hausdorff space $X$, then the groupoid of germs $S \times_{\varphi} X$ is defined as follows (see for example [10, 23]). Define a relation $\sim$ on $\left\{(s, x) \in S \times X: x \in \operatorname{dom}\left(\varphi_{s}\right)\right\}$ by $(s, x) \sim\left(s^{\prime}, y\right)$ if $x=y$ and there is an idempotent $p \in E(S)$ such that $x \in \operatorname{dom}(p)$ and $s p=s^{\prime} p$. This is an equivalence relation, and the collection $S \times{ }_{\varphi} X$ of equivalence classes for this relation is a locally compact étale groupoid with unit space $X$ and structure maps $r([s, x])=\varphi_{s}(x), s([s, x])=x,\left[s, \varphi_{t}(x)\right][t, x]=[s t, x]$, and $[s, x]^{-1}=\left[s^{*}, \varphi_{s}(x)\right]$. Moreover, if $\tilde{c}: S \backslash\{0\} \rightarrow \Gamma$ is a grading, then, as idempotent elements $p \in S \backslash\{0\}$ satisfy $\tilde{c}(p)=e$, there is a grading of $S \times_{\varphi} X$ given by $[s, x] \mapsto \tilde{c}(s)$. 
Proposition 5.4 of [10] implies that for graded ample Hausdorff groupoids $\mathcal{G}$, the groupoid of germs for the action $\theta$ of $S_{\mathcal{G}, \star}$ on $\mathcal{G}^{(0)}$ is canonically isomorphic to $\mathcal{G}$. Combining the preceding subsections with this result, we recover $\mathcal{G}$ from $N_{\star}(D)$ and $D$.

Lemma 3.10. Let $\mathcal{G}$ be an ample Hausdorff groupoid, let $c: \mathcal{G} \rightarrow \Gamma$ be a grading of $\mathcal{G}$ by a discrete group with $c^{-1}(e)$ topologically principal, let $R$ be a commutative integral domain with 1 , and let $N_{\star}(D) / \sim$ be the inverse semigroup of Lemma 3.8. Then there is an action $\varphi$ of $N_{\star}(D) / \sim$ on $\widehat{D}$ such that $\operatorname{dom}([f])=\left\{\pi: \pi\left(1_{s(\operatorname{supp}(f))}\right)=1\right\}, \operatorname{cod}([f])=$ $\left\{\pi: \pi\left(1_{r(\operatorname{supp}(f))}\right)=1\right\}$, and

$$
\varphi_{[f]}(\pi)(p)=\pi\left(f^{*} p f\right) \quad \text { for all } f \in N_{\star}(D) / \sim, \pi \in \operatorname{dom}([f]) \text { and } p \in E(D) .
$$

Moreover, if $\tilde{q}: N_{\star}(D) / \sim \rightarrow S_{\mathcal{G}, \star}$ is the isomorphism of Lemma 3.8, then the homeomorphism $\varepsilon: \mathcal{G}^{(0)} \rightarrow \widehat{D}$ intertwines $\theta_{\tilde{q}([f])}$ and $\varphi_{[f]}$ for every $f \in S$.

Proof. Lemma 3.9 shows that the formula given for $\varphi$ satisfies

$$
\varphi_{[f]}\left(\varepsilon_{u}\right)=\varepsilon_{\theta_{\tilde{q}([f])}}
$$

for all $f$ and $u$, so the result follows by pulling the action $\theta$ back to an action of $N_{\star}(D) / \sim$ via the isomorphism $\tilde{q}$.

We now obtain our key result.

Corollary 3.11. Let $\mathcal{G}$ be an ample Hausdorff groupoid, $c: \mathcal{G} \rightarrow \Gamma$ be a grading of $\mathcal{G}$ by a discrete group with $c^{-1}(e)$ topologically principal, $R$ be a commutative integral domain with 1 , and $N_{\star}(D) / \sim$ be the inverse semigroup of Lemma 3.8. Let $\varphi: N_{\star}(D) / \sim \curvearrowright \widehat{D}$ be the action of Lemma 3.10. Then, there is an isomorphism $\pi:\left(N_{\star}(D) / \sim\right) \times_{\varphi} \widehat{D} \rightarrow \mathcal{G}$ such that

$$
\pi\left(\left[[f], \varepsilon_{s(\alpha)}\right]\right)=\alpha
$$

for all $f \in N_{\star}(D)$ and $\alpha \in \operatorname{supp}(f)$. Moreover, $\pi$ intertwines the grading on $\left(N_{\star}(D) / \sim\right) \times_{\varphi}$ $\widehat{D}$ induced by $\tilde{c}$ and the grading $c$ of $\mathcal{G}$.

Proof. Proposition 5.4 of [10] implies that there is an isomorphism

$$
\pi_{0}: S_{\mathcal{G}, \star} \times{ }_{\theta} \mathcal{G}^{(0)} \rightarrow \mathcal{G}
$$

such that $\pi_{0}([V, s(\alpha)])=\alpha$ for all $V \in S_{\mathcal{G}, \star}$ and $\alpha \in V$. This $\pi_{0}$ clearly carries the grading of $S_{\mathcal{G}, \star} \times{ }_{\theta} \mathcal{G}^{(0)}$ induced by $c$ to $c$. The final statement of Lemma 3.10 shows that $\tilde{q}$ and $\varepsilon$ induce an isomorphism of the action of $N_{\star}(D) / \sim$ on $\widehat{D}$ with that of $S_{\mathcal{G}, \star}$ on $\mathcal{G}^{(0)}$. Thus, there is an isomorphism $\pi^{\prime}:\left(N_{\star}(D) / \sim\right) \times_{\varphi} \widehat{D} \rightarrow S_{\mathcal{G}, \star} \times_{\theta} \mathcal{G}^{(0)}$ such that $\pi^{\prime}\left(\left[[f], \varepsilon_{u}\right]\right)=[\tilde{q}(f), u]$ for all $f$ and $u$, and $\pi^{\prime}$ takes the grading of $\left(N_{\star}(D) / \sim\right) \times_{\varphi} \widehat{D}$ induced by $\tilde{c}$ to the grading of $S_{\mathcal{G}, \star} \times{ }_{\theta} \mathcal{G}^{(0)}$ induced by $c$. Therefore, $\pi=\pi_{0} \circ \pi^{\prime}$ is the desired isomorphism.

Remark 3.12. As an alternative to Corollary 3.11, one might aim to employ LawsonLenz's noncommutative generalisation of Stone duality [20, Theorem 3.25] (see also [19]) to recover $\mathcal{G}$ from $N_{\star}(D) / \sim$. Indeed, as the anonymous referee points out, the results of this section could possibly be reformulated as a statement of graded duality, between appropriate categories of graded boolean inverse semigroups and graded étale groupoids. However, we will not pursue this interpretation further here. 


\subsection{Proof of Theorem 3.1. We can now prove our main theorem.}

Proof of Theorem 3.1. Clearly if $\bar{\rho}: \mathcal{G} \rightarrow \mathcal{H}$ is a graded isomorphism of groupoids, then there is a graded isomorphism $\rho: A_{R}(\mathcal{G}) \rightarrow A_{R}(\mathcal{H})$ given by $\rho(f)=f \circ \bar{\rho}^{-1}$. Moreover, this isomorphism carries $D_{\mathcal{G}}$ to $D_{\mathcal{H}}$ because $\bar{\rho}$ carries $\mathcal{G}^{(0)}$ to $\mathcal{H}^{(0)}$.

For the reverse implication, suppose that $\rho: A_{R}(\mathcal{G}) \rightarrow A_{R}(\mathcal{H})$ is a graded ring isomorphism with $\rho\left(D_{\mathcal{G}}\right) \subseteq D_{\mathcal{H}}$. Lemma 2.1 applied to $c^{-1}(e)$ implies that $D_{\mathcal{G}}$ is maximal abelian subring of $A_{R}(\mathcal{G})_{e}$, which implies that $\rho\left(D_{\mathcal{G}}\right)$ is a maximal abelian subring of $A_{R}(\mathcal{H})_{e}$ because $\rho$ is a graded ring isomorphism. Since $\rho\left(D_{\mathcal{G}}\right)$ is contained in the abelian subring $D_{\mathcal{H}}$ of $A_{R}(\mathcal{H})_{e}$, we deduce that $\rho\left(D_{\mathcal{G}}\right)=D_{\mathcal{H}}$. Thus, $\rho$ restricts to an isomorphism of boolean rings $E\left(D_{\mathcal{G}}\right) \cong E\left(D_{\mathcal{H}}\right)$ inducing a homeomorphism $\rho^{*}: \widehat{D}_{\mathcal{H}} \cong \widehat{D}_{\mathcal{G}}$ given by $\rho^{*}(\pi)=\pi \circ \rho$.

If $n$ is a homogeneous graded normaliser of $D_{\mathcal{G}}$ in $A_{R}(\mathcal{G})$, then $\rho(n)$ is a homogeneous normaliser of $D_{\mathcal{H}}$ in $A_{R}(\mathcal{H})$ : the conditions defining a normaliser involve only the ring structure. Corollary 3.7 shows that $N_{\star}\left(D_{\mathcal{G}}\right)$ is an inverse semigroup, as is $N_{\star}\left(D_{\mathcal{H}}\right)$. Thus, $\rho$ restricts to a graded isomorphism $N_{\star}\left(D_{\mathcal{G}}\right) \cong N_{\star}\left(D_{\mathcal{H}}\right)$ of inverse semigroups. Since $\rho$ carries $E\left(D_{\mathcal{G}}\right)$ to $E\left(D_{\mathcal{H}}\right)$, the equivalence relations $\sim$ on $N_{\star}\left(D_{\mathcal{G}}\right)$ and $N_{\star}\left(D_{\mathcal{H}}\right)$ defined in Lemma 3.8 satisfy $f \sim h$ if and only if $\rho(f) \sim \rho(h)$. So $\rho$ induces a graded isomorphism $\tilde{\rho}: N_{\star}\left(D_{\mathcal{G}}\right) / \sim \rightarrow N_{\star}\left(D_{\mathcal{H}}\right) / \sim$. The definition of $\rho^{*}$ in the preceding paragraph and the formula (3.5) in Lemma 3.10 show that $\rho^{*}\left(\varphi_{\tilde{\rho}([f])}(\pi)\right)=\varphi_{[f]}\left(\rho^{*}(\pi)\right)$ for all $f \in N_{\star}\left(D_{\mathcal{G}}\right)$ and $\pi \in \widehat{D}_{\mathcal{H}}$. So there exists a graded isomorphism

$$
\hat{\rho}:\left(N_{\star}\left(D_{\mathcal{G}}\right) / \sim\right) \times_{\varphi} \widehat{D}_{\mathcal{G}} \cong\left(N_{\star}\left(D_{\mathcal{H}}\right) / \sim\right) \times_{\varphi} \widehat{D}_{\mathcal{H}}
$$

that carries $\left[[f], \rho^{*}(\pi)\right]$ to $[[\rho(f)], \pi]$ for $f \in N_{\star}\left(D_{\mathcal{G}}\right)$ and $\pi \in \widehat{D}_{\mathcal{H}}$. Now the graded isomorphisms $\pi_{\mathcal{G}}:\left(N_{\star}\left(D_{\mathcal{G}}\right) / \sim\right) \times_{\varphi} \widehat{D}_{\mathcal{G}} \rightarrow \mathcal{G}$ and $\pi_{\mathcal{H}}:\left(N_{\star}\left(D_{\mathcal{H}}\right) / \sim\right) \times_{\varphi} \widehat{D}_{\mathcal{H}} \rightarrow \mathcal{H}$ yield a graded isomorphism $\bar{\rho}:=\pi_{\mathcal{H}} \circ \hat{\rho} \circ \pi_{\mathcal{G}}^{-1}: \mathcal{G} \rightarrow \mathcal{H}$.

Remark 3.13. It is worth discussing the extent to which the hypotheses on our main theorem are necessary.

- If $c^{-1}(\mathcal{G})_{e}$ is not topologically principal, then $D_{\mathcal{G}}$ is not necessarily maximal abelian in $A_{R}(\mathcal{G})_{e}$; and, in addition, key steps in our analysis of the normalisers of $D_{\mathcal{G}}$ and of the quotient $N_{\star}\left(D_{\mathcal{G}}\right) / \sim$ break down. It is not clear, however, that this hypothesis is necessary to our main result: Brown-Clark-an Huef [6] show that the conclusion of our main theorem is valid for arbitrary graph groupoids with the trivial grading. Our theorem also holds in the special case that $\mathcal{G}$ has one unit (is a discrete group), $c$ is the trivial grading and $R=\mathbb{Z}$ : it then reduces to the classical fact that the group-ring construction and the group-of-units construction are adjoint functors.

- It is unclear whether it is necessary to assume that $R$ is an integral domain or that it is unital. These hypotheses are used heavily in our analysis, but we do not have a counterexample to our main result in their absence.

- It is, however, necessary to make some assumptions on $R$ : Let $R:=C_{\mathrm{lc}}(K)$, the ring of locally-constant complex-valued functions on the Cantor set. Since $K \cong K \sqcup K$, we have $R \cong R \oplus R$. Hence, for any ample Hausdorff groupoid $\mathcal{G}$, there exists a diagonal-preserving isomorphism $A_{R}(\mathcal{G}) \cong A_{R}(\mathcal{G}) \oplus A_{R}(\mathcal{G})=A_{R}(\mathcal{G} \sqcup \mathcal{G})$, whereas $\mathcal{G}$ and $\mathcal{G} \sqcup \mathcal{G}$ are not usually isomorphic. 


\section{Applications}

4.1. Topologically principal groupoids. Here we record what our results say for ungraded ample groupoids $\mathcal{G}$. Given any groupoid $\mathcal{G}$ we can endow it with the trivial grading $c: \mathcal{G} \rightarrow\{e\}$, and then apply our main theorem. In this instance, we have $N_{\star}(D)=N(D)$ and $C_{\mathrm{lc}}\left(\mathcal{G}, R^{\times} \cup\{0\}\right)_{\star}=C_{\mathrm{lc}}\left(\mathcal{G}, R^{\times} \cup\{0\}\right)$.

Theorem 4.1. Let $\mathcal{G}$ be an ample Hausdorff groupoid and suppose that $\mathcal{G}$ is topologically principal. Let $R$ be a commutative integral domain with 1 . Then

(1) $N(D)=\left\{n \in A_{R}(\mathcal{G}): \operatorname{supp}(n)\right.$ is a local bisection and $\left.n(\mathcal{G}) \subseteq R^{\times} \cup\{0\}\right\}$.

(2) For each $n \in N(D)$, there is a unique $n^{*} \in A_{R}(\mathcal{G})$ such that $n D n^{*} \cup n^{*} D n \subseteq D$, $n n^{*} n=n$ and $n^{*} n n^{*}=n^{*}$. Moreover, the element $n^{*}$ belongs to $N(D)$, and $N(D)$ is an inverse semigroup under* .

(3) There is an equivalence relation on $N(D)$ given by $n_{1} \sim n_{2}$ if and only if $n_{1}^{*} p n_{1}=$ $n_{2}^{*} p n_{2}$ and $n_{1} p n_{1}^{*}=n_{2} p n_{2}^{*}$ for every idempotent $p \in D$. Furthermore, $N(D) / \sim$ is an inverse semigroup under the operations inherited from $N(D)$.

(4) There is an action $\varphi$ of $N(D) / \sim$ on the Stone spectrum $\widehat{D}$ of the collection of idempotent elements of $D$ such that $\varphi_{[n]}(\pi)(p)=\pi\left(n^{*} p n\right)$ for all $n \in N(D), \pi \in \widehat{D}$ and every idempotent $p \in D$.

(5) There is an isomorphism $\mathcal{G} \cong(N(D) / \sim) \times_{\varphi} \widehat{D}$ that carries $\alpha \in \mathcal{G}$ to $\left[\left[1_{V}\right], \varepsilon_{s(\alpha)}\right]$ for any compact open local bisection $V$ containing $\alpha$.

Proof. Let $c: \mathcal{G} \rightarrow\{e\}$ be the trivial grading. Corollary 3.7 applied to this grading $c$ gives (1) and (2). Part (3) follows from Lemma 3.8 with the trivial grading, and (4) follows from Lemma 3.10. Finally, the inverse of the isomorphism $\pi$ obtained from Corollary 3.11 satisfies the formula described in (5).

Remark 4.2. The action of $N(D)$ on $\widehat{D}$ given by $(n \cdot \pi)(e)=\pi\left(n^{*} e n\right)$, which induces an action of $N(D) / \sim$ in Theorem 4.1(4) is usually called the spectral action, and it enjoys a universal property (see [27] for more details). In particular, as described in [27, Example 5.9], this action is the dual of the (right) Munn representation.

Moreover, the isomorphism described in Theorem 4.1(4) induces the isomorphism of Steinberg algebras $A_{R}\left(N(D) / \sim \times_{\varphi} \hat{D}\right) \cong A_{R}(\mathcal{G})$ described in [27, Theorem 6.3]; thus, Theorem 4.1 is, in a sense, dual to Steinberg's result.

Corollary 4.3. Let $\mathcal{G}$ and $\mathcal{H}$ be ample Hausdorff topologically principal groupoids and let $R$ be a commutative integral domain with 1. The following are equivalent:

(1) The groupoids $\mathcal{G}$ and $\mathcal{H}$ are isomorphic as topological groupoids.

(2) There is ring isomorphism $\rho: A_{R}(\mathcal{G}) \rightarrow A_{R}(\mathcal{H})$ such that $\rho\left(D_{\mathcal{G}}\right) \subseteq D_{\mathcal{H}}$.

Proof. Apply Theorem 3.1 to the trivial grading $\Gamma: \mathcal{G} \rightarrow\{e\}$.

4.2. Ring-isomorphisms of Leavitt path algebras. In this short section, we make an observation about the implications of our results for Leavitt path algebras. For background on Leavitt path algebras and on Abrams and Tomforde's isomorphism conjecture, see [1, 2, 15, 28, 29].

Abrams and Tomforde conjectured that if $E$ and $F$ are graphs for which there is a ring isomorphism $L_{R}(E) \cong L_{R}(F)$ for some $\operatorname{ring} R$, then $C^{*}(E) \cong C^{*}(F)$ as $C^{*}$-algebras. This conjecture remains open, but we make some headway (see also [6] and Remark 4.5 below). 
Corollary 4.4. Suppose that $E$ and $F$ are graphs in which every cycle has an exit. Then the following are equivalent:

(1) There exists a commutative integral domain $R$ with 1 for which there is an isomorphism $\pi: L_{R}(E) \rightarrow L_{R}(F)$ satisfying $\pi\left(s_{\mu} s_{\mu^{*}}\right) \in \operatorname{span}_{R}\left\{s_{\eta} s_{\eta^{*}}: \eta \in F^{*}\right\}$ for all $\mu \in E^{*}$.

(2) There exists a commutative integral domain $R$ with 1 for which there is an isomorphism $\pi: L_{R}(E) \rightarrow L_{R}(F)$ satisfying $\pi\left(s_{\mu} s_{\mu^{*}}\right) s_{\eta} s_{\eta^{*}}=s_{\eta} s_{\eta^{*}} \pi\left(s_{\mu} s_{\mu^{*}}\right)$ for all $\mu \in E^{*}$ and $\eta \in F^{*}$.

(3) For every ${ }^{*}$-ring $S$ there exists $a^{*}{ }^{*}$-isomorphism of $L_{S}(E)$ onto $L_{S}(F)$ that carries $\operatorname{span}_{S}\left\{s_{\mu} s_{\mu^{*}}: \mu \in E^{*}\right\}$ to $\operatorname{span}_{S}\left\{s_{\eta} s_{\eta^{*}}: \eta \in F^{*}\right\}$.

(4) There is an isomorphism $\psi: C^{*}(E) \rightarrow C^{*}(F)$ such that $\psi\left(\overline{\operatorname{span}}\left\{s_{\mu} s_{\mu}^{*}: \mu \in E^{*}\right\}\right)=$ $\overline{\operatorname{span}}\left\{s_{\eta} s_{\eta}^{*}: \eta \in F^{*}\right\}$.

(5) There is an isomorphism $\psi: C^{*}(E) \rightarrow C^{*}(F)$ such that $\psi\left(s_{\mu} s_{\mu}^{*}\right) s_{\eta} s_{\eta}^{*}=s_{\eta} s_{\eta}^{*} \psi\left(s_{\mu} s_{\mu}^{*}\right)$ for all $\mu \in E^{*}$ and $\eta \in F^{*}$.

Recall from [9, Example 3.2] that if $E$ is a directed graph, then there is an isomorphism

$$
\alpha_{E}: L_{R}(E) \cong A_{R}\left(\mathcal{G}_{E}\right)
$$

that carries $\operatorname{span}_{S}\left\{s_{\mu} s_{\mu^{*}}: \mu \in E^{*}\right\}$ to $D_{\mathcal{G}_{E}}$. We will use this isomorphism at a number of points in the proof of Corollary 4.4.

Proof of Corollary 4.4. It is well known (see [17]) that the groupoid $\mathcal{G}_{E}$ of a directed graph $E$ is topologically principal provided that every cycle in $E$ has an exit. So $\mathcal{G}_{E}$ and $\mathcal{G}_{F}$ are topologically principal.

We first prove (11) $\Longleftrightarrow$ (2). The implication (11) implies (2) is trivial. For the reverse, observe that if $\pi$ is as in (2), then each $\pi\left(s_{\mu} s_{\mu^{*}}\right)$ commutes with every element of $\operatorname{span}_{R}\left\{s_{\eta} s_{\eta^{*}}: \eta \in F^{*}\right\}$. Since the latter is a maximal abelian subring by Lemma 2.1, it follows that each $\pi\left(s_{\mu} s_{\mu^{*}}\right) \in \operatorname{span}_{R}\left\{s_{\eta} s_{\eta^{*}}: \eta \in F^{*}\right\}$.

Next we prove that (11) implies (13) and (5). Suppose that (1) holds. Corollary 4.3 implies that the graph groupoids $\mathcal{G}_{E}$ and $\mathcal{G}_{F}$ are isomorphic; say $\rho: \mathcal{G}_{F} \rightarrow \mathcal{G}_{E}$ is an isomorphism. Then $\rho$ restricts to a homeomorphism $\mathcal{G}_{F}^{(0)} \rightarrow \mathcal{G}_{E}^{(0)}$. For each $*$-ring $S, \rho$ induces a $*-$ isomorphism $\rho^{*}: A_{S}\left(\mathcal{G}_{E}\right) \rightarrow A_{S}\left(\mathcal{G}_{F}\right)$ satisfying $\rho^{*}(f)=f \circ \rho$. In particular, $\rho$ carries $D_{\mathcal{G}_{E}} \subseteq A_{S}\left(\mathcal{G}_{E}\right)$ to $D_{\mathcal{G}_{F}} \subseteq A_{S}\left(\mathcal{G}_{F}\right)$. So $\alpha_{F}^{-1} \circ \rho^{*} \circ \alpha_{E}$ is a $*$-isomorphism $L_{S}(E) \rightarrow L_{S}(F)$ as required in (3). Similarly the isomorphism $\rho$ induces a $\mathrm{C}^{*}$-algebra isomorphism $\rho^{*}$ : $C^{*}\left(\mathcal{G}_{E}\right) \rightarrow C^{*}\left(\mathcal{G}_{F}\right)$ satisfying $\rho^{*}(f)=f \circ \rho$ for $f \in C_{c}\left(\mathcal{G}_{E}\right)$. In particular $\rho^{*}\left(C_{0}\left(\mathcal{G}_{E}^{(0)}\right)\right)=$ $C_{0}\left(\mathcal{G}_{F}^{(0)}\right)$. It is standard that there is an isomorphism $\phi_{E}: C^{*}(E) \rightarrow C^{*}\left(\mathcal{G}_{E}\right)$ that carries $\overline{\operatorname{span}}\left\{s_{\mu} s_{\mu}^{*}: \mu \in E^{*}\right\}$ to $C_{0}\left(\mathcal{G}_{E}^{(0)}\right)$, and similarly a diagonal-preserving isomorphism $\phi_{F}$ : $C^{*}(F) \rightarrow C^{*}\left(\mathcal{G}_{F}\right)$. So $\psi:=\phi_{F}^{-1} \circ \rho^{*} \circ \phi_{E}$ is the isomorphism in (15).

Now we prove (3) implies (1). Suppose that (3) holds. Taking $S=\mathbb{F}_{2}$ (the field of two elements), for example, trivially gives (1).

For (5) implies (1), suppose that (5) holds. With $\phi_{E}$ and $\phi_{F}$ as above, the map $\phi_{F} \circ$ $\psi \circ \phi_{E}^{-1}$ is an isomorphism $C^{*}\left(\mathcal{G}_{E}\right) \rightarrow C^{*}\left(\mathcal{G}_{F}\right)$ that carries the Cartan subalgebra $C_{0}\left(\mathcal{G}_{E}^{(0)}\right)$ to the Cartan subalgebra $C_{0}\left(\mathcal{G}_{F}^{(0)}\right)$. So [26, Proposition 4.13] implies that there is an isomorphism $\rho: \mathcal{G}_{F} \cong \mathcal{G}_{E}$ as in the first paragraph. This induces an isomorphism $\rho^{*}$ : $A_{R}\left(\mathcal{G}_{E}\right) \rightarrow A_{R}\left(\mathcal{G}_{F}\right)$ that takes $D_{\mathcal{G}_{E}}$ to $D_{\mathcal{G}_{F}}$. So $\alpha_{F}^{-1} \circ \rho^{*} \circ \alpha_{E}$ is the desired isomorphism of Leavitt path algebras. 
It remains to prove (44) $\Longleftrightarrow$ (5). The implication (44) implies (5) is trivial; and (5) implies (4) by the same argument as we used for (2) implies (11) because Renault's theorems prove that $\overline{\operatorname{span}}\left\{s_{\eta} s_{\eta}^{*}: \eta \in F^{*}\right\}$ is a maximal abelian subalgebra of $C^{*}(F)$.

Remark 4.5. We learned of the paper [6] in the later stages of the preparation of this manuscript. Our Corollary 4.4 is related to the main theorem [6, Theorem 5.3], though neither strictly generalises the other. There are two differences between the two results:

- Theorem 5.3 of [6] applies to row-finite graphs $E$ and $F$ with no sinks, whereas our result applies to arbitrary graphs $E$ and $F$ in which every cycle has an exit.

- The hypotheses of [6, Theorem 5.3] demand that the isomorphism $\pi: L_{R}(E) \rightarrow$ $L_{R}(F)$ should be a ${ }^{*}$-isomorphism, and that it should restrict to an isomorphism $\pi: D_{E} \rightarrow D_{F}$; whereas Corollary 4.4 requires only a ring isomorphism $L_{R}(E) \rightarrow$ $L_{R}(F)$ that carries $D_{E}$ into the commutant of $D_{F}$.

We use our results to obtain an improvement of [13, Theorem 3.6]. For its statement, we need some standard graph-theoretical definitions, as follows.

Definition 4.6. A graph $E$ is said to be:

(1) strongly connected if there is a path between any two vertices.

(2) essential if it has no sinks or sources, and

(3) trivial if it is a single cycle with no other edges or vertices.

Corollary 4.7. Let E, F be finite, essential, non-trivial, strongly connected graphs, and let $R$ be any commutative integral domain with 1 . If there is an isomorphism $\phi: L_{R}(E) \rightarrow$ $L_{R}(F)$ such that $\phi\left(\mathcal{D}\left(L_{R}(E)\right)\right) \subseteq \mathcal{D}\left(L_{R}(F)\right)$, then

$$
\operatorname{sgn}\left(\operatorname{det}\left(I-A_{E}\right)\right)=\operatorname{sgn}\left(\operatorname{det}\left(I-A_{F}\right)\right) .
$$

Proof. It is straightforward to check that the conditions on $E$ and $F$ imply that both graphs have the property that every cycle has an exit. By Corollary 4.4, we obtain a $C^{*}$ algebra isomorphism $\bar{\phi}: C^{*}(E) \rightarrow C^{*}(F)$ such that $\bar{\phi}\left(\mathcal{D}\left(C^{*}(E)\right)\right)=\mathcal{D}\left(C^{*}(F)\right)$. It follows from [13, Theorem 3.3] (cf. [22, Theorem 3.6]) that $\operatorname{sgn}\left(\operatorname{det}\left(I-A_{E}\right)\right)=\operatorname{sgn}(\operatorname{det}(I-$ $\left.\left.A_{F}\right)\right)$.

This result can be applied to give a partial answer to one of the most intriguing open questions in the theory of Leavitt path algebras; namely, whether, for a commutative coefficient ring $R$ with 1 , the algebras $L_{2, R}$ and $L_{2-, R}$ are isomorphic. Johansen and Sørensen have recently shown that there is no $*$-isomorphism between $L_{2, \mathbb{Z}}$ and $L_{2-, \mathbb{Z}}$ ([13]). Recall from e.g. [13] that $L_{2, R}$ denotes the classical Leavitt algebra of type $(1,2)$ with coefficients in $R$. It is the Leavitt path $R$-algebra of the graph $E_{2}$ with one vertex and two arrows. The algebra $L_{2-, R}$ is the Leavitt path $R$-algebra associated to a graph $E_{2-}$ depicted in the introduction to [13]. Over any regular supercoherent coefficient ring $R$, both algebras $L_{2, R}$ and $L_{2-, R}$ have trivial algebraic $K$-theory ([3]). However, they are distinguished by the numbers appearing in Corollary 4.7 .

Corollary 4.8. Let $R$ be a commutative integral domain with 1 . Then there is no isomorphism $\phi: L_{2, R} \rightarrow L_{2-, R}$ such that $\phi\left(\mathcal{D}\left(L_{2, R}\right)\right) \subseteq \mathcal{D}\left(L_{2-, R}\right)$ or $\phi^{-1}\left(\mathcal{D}\left(L_{2-, R}\right)\right) \subseteq \mathcal{D}\left(L_{2, R}\right)$.

Proof. Assume there is an isomorphism $\phi: L_{2, R} \rightarrow L_{2-, R}$ such that $\phi\left(\mathcal{D}\left(L_{2, R}\right)\right) \subseteq \mathcal{D}\left(L_{2-, R}\right)$ or $\phi^{-1}\left(\mathcal{D}\left(L_{2-, R}\right)\right) \subseteq \mathcal{D}\left(L_{2, R}\right)$. The graphs $E_{2}$ and $E_{2-}$ are finite, essential, non-trivial and strongly connected. Therefore, it follows from Corollary 4.7 that

$$
\operatorname{sgn}\left(\operatorname{det}\left(I-A_{E_{2}}\right)\right)=\operatorname{sign}\left(\operatorname{det}\left(I-A_{E_{2-}}\right)\right) \text {. }
$$


However $\operatorname{det}\left(I-A_{E_{2}}\right)=-1$ and $\operatorname{det}\left(I-A_{E_{2-}}\right)=+1$, so we obtain a contradiction.

4.3. Graded ring-isomorphisms of Kumjian-Pask algebras. In this section, we emphasise what extra information we obtain by keeping track of the graded structure in Section 3. Recall that for every $k$-graph $\Lambda$, the associated $k$-graph groupoid $\mathcal{G}_{\Lambda}$ (see [15] or [12]) is $\mathbb{Z}^{k}$-graded, and $c^{-1}(0)$ is a principal groupoid. So our main theorem yields the following:

Corollary 4.9. Suppose that $\Lambda$ and $\Gamma$ are $k$-graphs and that $R$ is a commutative integral domain with 1. There is a graded ring-isomorphism $\phi: \mathrm{KP}_{R}(\Lambda) \cong \mathrm{KP}_{R}(\Gamma)$ such that $\phi\left(s_{\mu} s_{\mu^{*}}\right) s_{\eta} s_{\eta^{*}}=s_{\eta} s_{\eta^{*}} \phi\left(s_{\mu} s_{\mu^{*}}\right)$ for all $\mu \in \Lambda$ and $\eta \in \Gamma$ if and only if the groupoids $\mathcal{G}_{\Lambda}$ and $\mathcal{G}_{\Gamma}$ are isomorphic, in which case there is a diagonal preserving isomorphism $\mathrm{KP}_{S}(\Lambda) \cong \mathrm{KP}_{S}(\Gamma)$ for every ring $S$, and there is a diagonal-preserving isomorphism $C^{*}(\Lambda) \cong C^{*}(\Gamma)$.

Proof. The argument is essentially the same as the corresponding implications in Corollary 4.4, except that we apply Theorem 3.1 instead of Corollary 4.3.

\section{REFERENCES}

[1] G. Abrams and G. Aranda Pino, The Leavitt path algebra of a graph, J. Algebra 293 (2005), 319-334.

[2] G. Abrams and M. Tomforde, Isomorphism and Morita equivalence of graph algebras, Trans. Amer. Math. Soc. 363 (2011), 3733-3767.

[3] P. Ara, M. Brustenga, G. Cortiñas, K-theory of Leavitt path algebras, Münster Journal of Mathematics, 2 (2009), 5-34.

[4] P. Ara and R. Exel, Dynamical systems associated to separated graphs, graph algebras, and paradoxical decompositions, Adv. Math. 252 (2014), 748-804.

[5] P. Ara, M.A. Moreno and E. Pardo, Nonstable K-theory for graph algebras, Algebr. Represent. Theory 10 (2007), 157-178.

[6] J.H. Brown, L.O. Clark and A. an Huef, Diagonal-preserving ring ${ }^{*}$-isomorphisms of Leavitt path algebras, preprint 2015 (arXiv:1510.05309 [math.RA]).

[7] N. Brownlowe, T.M. Carlsen and M. F. Whittaker, Graph algebras and orbit equivalence, Ergodic Theory Dynam. Systems, to appear (arXiv:1410.2308 [math.OA]).

[8] L.O. Clark, C. Farthing, A. Sims and M. Tomforde, A groupoid generalisation of Leavitt path algebras, Semigroup Forum 89 (2014), 501-517.

[9] L.O. Clark and A. Sims, Equivalent groupoids have Morita equivalent Steinberg algebras, J. Pure Appl. Algebra 219 (2015), 2062-2075.

[10] R. Exel, Inverse semigroups and combinatorial $C^{*}$-algebras, Bull. Braz. Math. Soc. (N.S.) 39 (2008), 191-313.

[11] R. Exel, Partial dynamical systems, Fell bundles and applications, New York J. Math. book series, to appear (arXiv:1511.04565 [math.OA]).

[12] C. Farthing, P.S. Muhly and T. Yeend, Higher-rank graph $C^{*}$-algebras: an inverse semigroup and groupoid approach, Semigroup Forum 71 (2005), 159-187.

[13] R. Johansen and A.P.W. Sørensen, The Cuntz splice does not preserve *-isomorphism of Leavitt path algebras over $\mathbb{Z}$, J. Pure Appl. Algebra 220 (2016), 3966-3983.

[14] A. Kumjian, On $C^{*}$-diagonals, Canad. J. Math. 38 (1986), 969-1008.

[15] A. Kumjian and D. Pask, Higher rank graph $C^{*}$-algebras, New York J. Math. 6 (2000), 1-20.

[16] A. Kumjian, D. Pask and I. Raeburn, Cuntz-Krieger algebras of directed graphs, Pacific J. Math. 184 (1998), 161-174.

[17] A. Kumjian, D. Pask, I. Raeburn and J. Renault, Graphs, groupoids, and Cuntz-Krieger algebras, J. Funct. Anal. 144 (1997), 505-541.

[18] M.V. Lawson, Inverse semigroups, The theory of partial symmetries, World Scientific Publishing Co., Inc., River Edge, NJ, 1998, xiv+411. 
[19] M.V. Lawson, A noncommutative generalization of Stone duality, J. Aust. Math. Soc. 88 (2010), $385-404$.

[20] M.V. Lawson and D.H. Lenz, Pseudogroups and their étale groupoids, Adv. Math. 244 (2013), 117170 .

[21] X. Li, Continuous orbit equivalence rigidity, preprint 2015 (arXiv:1503.01704 [math.DS]).

[22] K. Matsumoto and H. Matui, Continuous orbit equivalence of topological Markov shifts and CuntzKrieger algebras, Kyoto J. Math. 54 (2014), 863-877.

[23] A.L.T. Paterson, Groupoids, inverse semigroups, and their operator algebras, Birkhäuser Boston Inc., Boston, MA, 1999, xvi+274.

[24] A.L.T. Paterson, Graph inverse semigroups, groupoids and their $C^{*}$-algebras, J. Operator Theory 48 (2002), 645-662.

[25] J. Renault, A groupoid approach to $C^{*}$-algebras, Springer, Berlin, 1980, ii +160 .

[26] J. Renault, Cartan subalgebras in $C^{*}$-algebras, Irish Math. Soc. Bulletin 61 (2008), 29-63.

[27] B. Steinberg, A groupoid approach to discrete inverse semigroup algebras, Adv. Math. 223 (2010), 689-727.

[28] M. Tomforde, Uniqueness theorems and ideal structure for Leavitt path algebras, J. Algebra 318 (2007), 270-299.

[29] M. Tomforde, Leavitt path algebras with coefficients in a commutative ring, J. Pure Appl. Algebra 215 (2011), 471-484.

[30] M. Tomforde (Ed.), The graph algebra problem page, http://www.math. uh.edu/ tomforde/GraphAlgebraProblems/GraphAlgebraProblemPage.html

[31] F. Wehrung, Refinement monoids, equidecomposability types, and Boolean inverse semigroups, 216 pp. 2016 (hal-01197354v2). https://hal.archives-ouvertes.fr/hal-01197354.

Department of Mathematics, Universitat Autońoma de Barcelona, 08193 Bellaterra (BARCELONA), Spain

E-mail address: para@mat.uab.cat

School of Mathematics and Statistics, University of Glasgow, 15 University Gardens, G12 8QW, Glasgow, United KingDom

E-mail address: joan.bosa@glasgow.ac.uk

Centre for Research in Mathematics, Western Sydney University, Australia

E-mail address: r.hazrat@westernsydney.edu.au

School of Mathematics and Applied Statistics, University of Wollongong, NSW 2522, Australia

E-mail address: asims@uow.edu.au 